\title{
Recensement du peuplement en fourmis d'un écosystème forestier méditerranéen soumis à une irradiation gamma chronique
}

\author{
G.H. PERRAULT*, R. CASTET**
}

RÉSUMÉ

\begin{abstract}
La population de fourmis d'un écosystème méditerranéen est recensée après 18 ans d'une irradiation chronique in situ par une source de ${ }^{137}$ Cs (44,4 TBq à l'origine). Les changements de population observés peuvent être rapportés aux modifications induites par l'irradiation dans tout l'écosystème. Trois espèces de fourmis, dont deux ne s'y trouvaient pas en 1970, se sont installées très près de la source, ce qui implique une certaine radiorésistance que l'on peut rapprocher de la résistance écologique naturelle de ces espèces.
\end{abstract}

D'un point de vue général, on peut enfin admettre que l'irradiation chronique agit comme un stress physique naturel tel que le froid ou la sécheresse.

\section{ABSTRACT}

The ant population of a mediterranean ecosystem was recorded 18 years after field chronic irradiation by a ${ }^{137} \mathrm{Cs}$ source $(44.4 \mathrm{TBq}$ at the origin). The population change observed may be compared with the irradiation changes induced in the whole ecosystem. Three species of ants, two of which were not present in 1970, were collected very near the source. This implies some radioresistance that may be assigned to the natural ecological resistance of the species.

From a general point of view, it can be admitted that chronic irradiation acts as a natural physical stress like cold or dryness.

Georges Le MASNE et Annie BONAVITA-COUGOURDAN [12] ont publié, en 1972, les résultats d'une première étude, effectuée en 1970 , sur le peuplement en fourmis d'un écosystème forestier méditerranéen soumis depuis 11 mois à une irradiation gamma chronique. Cette station est située au centre d'études nucléaires de Cadarache dans le vallon des Charbonniers constitué en réserve naturelle protégée. Commencée le 9 juillet 1969, l'irradiation s'est poursuivie depuis lors. La source radioactive est constituée de

* Service mixte de contrôle biologique (SMCB), Direction des Centres d'expérimentations nucléaires (DIRCEN), B.P. 16, 91310 Montlhéry. Adresse actuelle: Service de Santé des Armées, SPRA, 1 bis, rue du Lieutenant R. Batany, 92140 Clamart.

** Commissariat à l'énergie atomique, Institut de protection et de sûreté nucléaire, DERS, Service d'études et recherches sur l'environnement, B.P. 1, 13108 St-Paul-lez-Durance Cedex. 
césium 137, d'une activité de $4,44.10^{13} \mathrm{~Bq}$ à l'origine (1 $200 \mathrm{Ci}$ ) et par conséquent, de $3,145.10^{13} \mathrm{~Bq}$ en 1985 (850 Ci). Elle est placée au sommet d'un tube support à $3,5 \mathrm{~m}$ du sol et irradie dans toutes les directions. Elle est descendue à chaque pénétration des expérimentateurs sur le terrain. Le dispositif est implanté sur la partie basse du versant exposé à l'Ouest, à une dizaine de mètres du chemin qui marque le fond du thalweg.

Dans un premier temps, les recherches se focalisèrent sur des observations des différents composants de cet écosystème; citons, par exemple, les travaux de FABRIES [10], suivis par ceux de BATTESTI-MARCHESE [1] sur la phytocénose; ceux de BIGOT et al [4], POINSOT-BALAGUER [16], ROUGON [17] sur la faune et la microflore, ceux de SAAS et al [18], TABONE et POINSOT-BALAGUER [19] sur la décomposition de la litière et celui de CASTET [7] sur l'activité globale du sol.

Dans un deuxième temps, la préoccupation fut de définir la position et le rôle de l'irradiation dans l'écologie de ce vallon, afin de bien situer ce facteur au niveau de l'écosystème naturel soumis en permanence aux divers facteurs de l'environnement. Les conclusions de ces différentes publications se rejoignent pour affirmer que cet écosystème forestier a été perturbé sous l'effet des rayonnements ionisants. Les réponses observées peuvent être dues aux conséquences directes ou indirectes de l'irradiation. C'est ainsi que l'élimination des espèces radiosensibles provoque inévitablement un déséquilibre dans la biocénose. Un nouvel arrangement des différents groupes induit une nouvelle structure de la cénose qui tend à atteindre un nouvel état d'équilibre.

Les études qui s'effectuent actuellement doivent prendre en compte cette évolution. Le MASNE et BONAVITA-COUGOURDAN [12] analysent plus particulièrement le peuplement des fourmis dans cette zone. Les auteurs ont, à l'époque, repéré 27 sociétés de fourmis à l'aide d'un piquetage dont, actuellement, il ne subsiste rien. La zone investiguée s'étendait sur un rayon de 20 à $30 \mathrm{~m}$ autour de la source. Notre objectif a été de constater l'état du peuplement après 16 ans d'irradiation chronique.

\section{MÉTHODE}

Elle consiste à recenser les espèces de fourmis présentes à l'intérieur d'un rectangle de 27 par $50 \mathrm{~m}$ entourant la source, perpendiculaire au chemin qui marque sensiblement le fond du thalweg. Sur le plan dressé par FABRIES [10] (fig. 1), le rectangle est presqu'entièrement contenu à l'intérieur de l'isodose $5,8 \mathrm{mGy}^{-\mathrm{h}^{-1}}$ située en 1985 à environ $22 \mathrm{~m}$ de la source. Cette limite est calculée par application de la formule $I=1,46.10^{-10} C^{\star} E^{\star} P^{\prime} D^{2},\left(C=3,145 \times 10^{13} \mathrm{~Bq}\right.$ en $1985, E=0,662 \mathrm{MeV}$, $P=0,92, D$ en mètres).

On effectue l'inventaire des nids en déplaçant les pierres et les divers objets recouvrant le sol. Quelques individus qui courent sur le sol sont prélevés occasionnellement. On procède à un battage sur nappe des arbustes bordant la zone et on tamise la litière et les écorces prélevées à la base des arbres. Dans la mesure du possible, on remet en place les objets et les pierres mais il n'est pas possible d'affirmer que notre intrusion restera sans effet. Ce travail fut réalisé les 26 et 27 juillet 1985 entre 9 et 12 heures, puis entre 14 et 16 heures, par temps sec et chaud, très ensoleillé. Les individus sont prélevés en alcool pour identification ultérieure. 


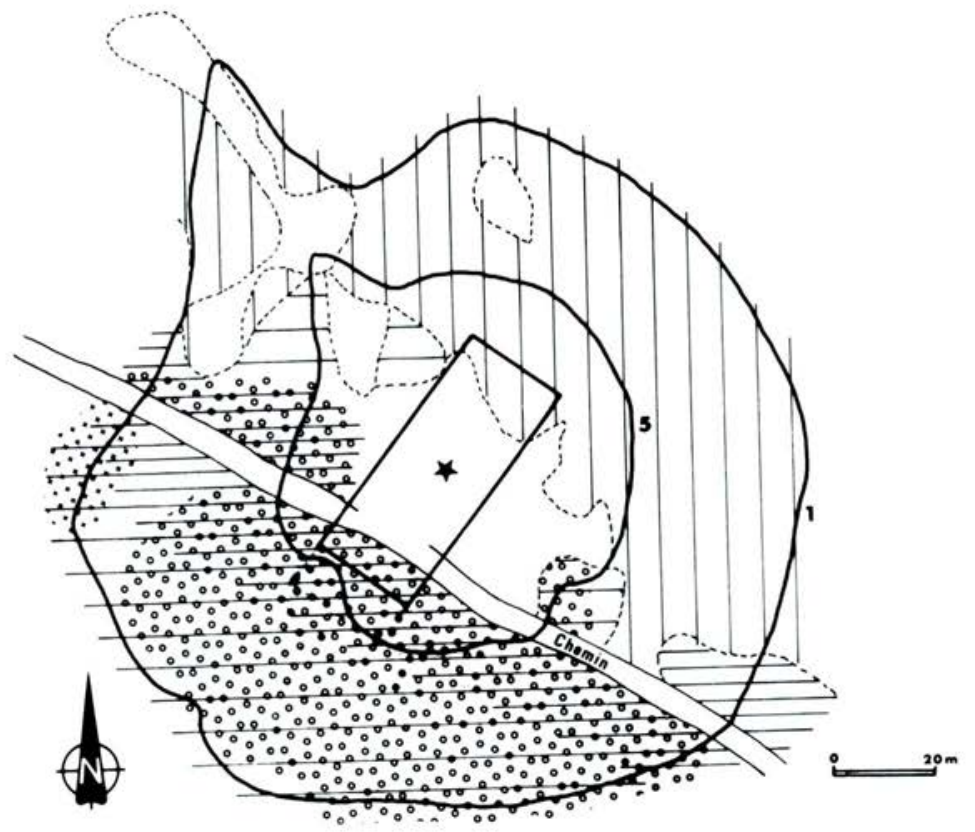

Fig. 1. - Végétation autour de la source du vallon des Charbonniers et isodoses en 1969, d'après FABRIES [10] (Les isodoses sont exprimées en mGy. ${ }^{-1}$ ).

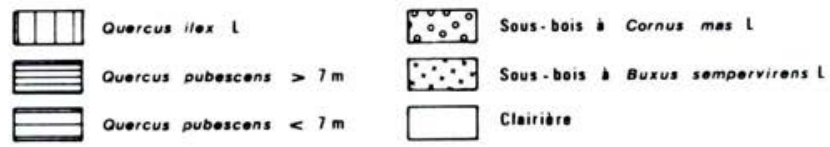

\section{RÉSULTATS}

\subsection{Inspection de la zone}

La zone prospectée se caractérise par quatre principaux types de végétation, qui définissent quatre phytocénoses différentes:

a) une clairière, située autour de l'irradiateur, qui se compose, à cette époque, et après 16 ans d'irradiation de quelques espèces herbacées (Rosa pimpinellifolia L. et Teucrium sp., et Hypericum perforatum L.);

b) une chênaie verte (Quercus ilex L.) en direction est-sud est, qui abrite essentiellement des taillis de Phyllirea sp.; 
c) une chênaie pubescente (Quercus pubescens) en direction ouest-nord ouest, avec des arbres qui atteignent une dizaine de mètres de hauteur, le sous-bois est fortement dominé par la présence de Cornus mas L.;

d) le thalweg à peuplement mixte avec une préférence pour le chêne pubescent.

II faut noter qu'à ces différents types de communautés végétales correspond, mis à part l'exposition, un micro-climat plus ou moins tamponné car la végétation, par son effet d'écran au rayonnement solaire, atténue les variations de température et d'humidité au niveau du sol. Un changement affectant la strate supérieure d'une phytocénose se répercutera sur les strates inférieures comme, par exemple, le sol et les organismes qui l'habitent.

L'évolution de ces zones sous irradiation a été analysée par BATTESTIMARCHESE [1]. La comparaison de la cartographie établie par FABRIES [10] et celle établie par cet auteur met en évidence l'étendue de la zone de létalité des ligneux. Tous ont régressé. Actuellement, ce sont les Phyllirea sp. (oléacées) qui sont les plus proches de la source; on les trouve aux environs de 9 à $10 \mathrm{~m}$, avec des malformations déjà décrites par FABRIES [10] (feuilles atteintes d'hypertrophie, couleur d'un vert plus intense, et parfois bilobées). Ils apparaissent souffrants avec les rameaux inférieurs couverts de lichen. Les cornouillers sont les plus éloignés de la source $(20 \mathrm{~m})$. Les premiers chênes pubescents de la bordure sud-ouest présentent une anomalie caractéristique d'un effet de masque, seules sont, en effet, vivantes les branches dont l'origine sur le tronc est opposée à la source. Celles-ci ne reçoivent que 50 à $70 \%$ de la dose à la même distance hors écran. Autour de l'irradiateur, la clairière s'est agrandie et appauvrie en arbustes (régression du romarin). Malgré l'importance des doses délivrées à cet endroit, la colonisation de cette station par les annuelles s'observe en dehors des périodes sèches, en particulier par une crucifère Cardamine hirsuta.

En conclusion, nous pouvons dire que cet écosystème s'est dégradé sous le stress de l'irradiation, c'est-à-dire que la variété des différentes communautés présentes avant l'irradiation s'est amoindrie, voire éteinte. II est certain que la régression des espèces végétales a bouleversé les maillons de l'écosystème qui leur étaient plus ou moins rattachés. On peut citer en exemple les maillons suivants:

- les espèces phytophages,

- les organismes peuplant la litière,

- le sol avec les êtres vivants qui recyclent la matière organique.

Sans oublier le rôle de la végétation comme régulateur climatique dans les strates inférieures. Le groupe des fourmis va, bien entendu, être affecté par ces changements.

D'assez nombreuses pierres de taille variable (depuis des galets de quelques centimètres de diamètre jusqu'à des parallélépipèdes de $25 \mathrm{~cm}$ d'arête) sont enfoncées en bordure du chemin et autour de la source. Ces pierres sont, pour la plupart, exposées au soleil dans la journée. Une importante surface centrale a été entourée d'un grillage et a servi à des expérimentations. Des conteneurs sont encore disposés sur le sol mais ils n'abritent aucune fourmi. La clairière est, en grande partie, une zone piétinée et remaniée. 


\subsection{Différents biotopes}

La prospection de la zone se fait en examinant les différents biotopes qui sont susceptibles d'abriter des fourmis; il faut noter qu'ils englobent un gradient d'irradiation. Dans cette étude nous en avons défini quatre, qui sont:

2.2.1. La clairière. La zone centrale, qui correspond à la partie la plus irradiée, et celle qui borde le chemin recèlent sous les pierres un certain nombre de fourmis (Tetramorium et Pheidole) en compagnie de quelques ténébrionides et carabiques. Une zone d'ombre s'étend en partie sur le chemin, côté futaie. De nombreux Tetramorium s'activent sous des galets fortement chauffés par le soleil. Un nid de Formica gagates Latreille est, par contre, situé sous une grosse pierre le plus souvent à l'ombre. Quelques grandes formicines parcourent la zone parmi lesquelles on prélève Cataglyphis cursor Fonscolombe et Formica gagates Latreille; quelques larves d'hémiptères courent à terre. Un nid de polistes contenu dans un pluviomètre couché sur le sol à $17 \mathrm{~m}$ de la source, comporte 20 alvéoles avec des larves bien vivantes. Les adultes s'enfuient à l'ouverture. Un deuxième nid de même taille est trouvé abandonné.

2.2.2. La lisière. Le battage de la végétation (filaires, cornouillers ou basses branches de chênes) permet la capture de Plagiolepis pygmaea Latreille du côté nord-est, et d'une faune variée au nord-est comme au sudouest comportant quelques coléoptères chrysomélides (une chrysomèle, quelques altises), un lyctus, des araignées (plusieurs espèces), des psoques ( 2 espèces) et des larves de chrysopidae. Cette récolte est faible, ce qui n'est pas surprenant compte tenu de la sécheresse et de la chaleur régnant sur ce genre de biotope, à cette période.

2.2.3. La litière. Examinée en bordure nord-est, sous les chênes pubescents, et sud-ouest, sous les chênes verts, la litière est sèche et recèle des fourmis également trouvées à découvert (Tetramorium caespitum (L.) et T. semilaeve André, Pheidole pallidula Nyl et Formica gagates Latreille) ou seulement présentes sous bois (Diplorhoptrum fugax (Latr.) s.l., Camponotus (Myrmentoma) piceus Leach, Leptothorax angustulus Nyl). Plagiolepis pygmaea Latreille est commun dans la litière et quelques individus fréquentent la strate arbustive de la lisière. On y trouve également quelques araignées, des coléoptères staphylins et des blattes.

2.2.4. Ecorces. Une forte population de Crematogaster scutellaris (Olivier) est cachée dans et sous les écorces de deux chênes pubescents situés à $22 \mathrm{~m}$ de la source. II peut s'agir d'une même société polycalique. Dolichoderus quadripunctatus L. est pris sous l'écorce d'un chêne voisin.

\subsection{Espèces recensées (figure 2).}

Tetramorium caespitum (L.) et Tetramorium semilaeve André (Tc et Ts).

Fourmis codominantes sous les pierres, dans la clairière, également présentes dans la litière, à l'est. Ces deux espèces sont taxonomiquement mal délimitées l'une par rapport à l'autre. Mais il y a ici deux formes très distinctes, une forme brune à forte striation, de grande taille, rattachable à la variété forte de $T$. caespitum (L.) et 
une forme distinctement plus petite, brun-jaunâtre à striation moins marquée qui peut être considérée comme $T$. semilaeve André. Les deux espèces ne sont pas présentes simultanément sous la même pierre. Un seul nid de $T$. caespitum (L.) contient du couvain; il est situé sous un galet de quelques centimètres d'épaisseur, exposé au soleil dès la fin de la matinée. Des galeries habitées, sous plusieurs pierres, témoignent d'une structure en réseau mais sans nid apparent. $T$. semilaeve André paraît ainsi nicher à la verticale de la source sous une pierre de $10 \mathrm{~cm}$ d'épaisseur, mais aucun Tetramorium ne circule à l'extérieur, ce qui n'est pas anormal étant donné la forte insolation régnant au centre de la clairière et qu'il s'agit d'une espèce à préférence nocturne.

\section{Pheidole pallidula (Nyl.) (PP).}

Codominante avec les Tetramorium, cette fourmi est présente sous des pierres au pied de la source, isolément ou en nombre et, dans toute la zone, d'ouest en est. Cette espèce construit des nids polycaliques dont les orifices de sortie peuvent déboucher aussi bien dans la clairière que dans la zone boisée de la lisière.

\section{Aphaenogaster subteranea (Lat.) (AS).}

La présence d'un unique mâle de cette espèce, aux prises avec des ouvrières de $T$. caespitum $L$., sous une pierre à $16 \mathrm{~m}$ de la source est vraisemblablement fortuite, aucune ouvrière n'étant visible aux alentours.

\section{Leptothorax angustulus (Nyl.) (LA).}

Une femelle désailée est accompagnée de quelques ouvrières dans la litière au pied d'un chêne pubescent de la bordure sud-ouest ( $23 \mathrm{~m}$ de la source). Le nid est vraisemblablement situé à cet endroit, dans une branchette de bois mort.

\section{Diplorhoptrum fugax (Latreille) s.l. (SF).}

Quelques ouvrières circulent dans la litière de la bordure sud-ouest. A cette époque de l'année, le nid est vraisemblablement sous terre, à peu de distance du lieu de capture des ouvrières.

\section{Crematogaster scutellaris (Olivier) (CS).}

De très nombreuses ouvrières sont dissimulées dans et sous des écorces au pied de deux chênes pubescents à environ $22 \mathrm{~m}$ de la source en bordure nord-ouest. Ce sont précisément ces chênes qui exhibent un effet de masque avec, pour seules branches vivantes, celles dont l'insertion est située à l'opposé de la source par rapport au tronc. Les Crematogaster sont, par contre, présents aussi bien de ce côté que face à la source.

\section{Dolichoderus quadripunctatus (L.) (DQ) - Camponotus (Myrmentoma) piceus Leach (CM).}

Ces deux espèces sont présentes dans la litière au pied d'un chêne pubescent. Les nids ne sont pas reperables, et ne sont pas nécessairement dans la zone prospectée.

\section{Plagiolepis pygmaea (Latreille) (Py).}

Quelques ouvrières circulent dans la litière du taillis nord-est et sur les filaires de la lisière nord-est. II est possible que cette espèce nidifie au printemps sous les pierres de la clairière et gagne la forêt en été comme l'a observé DU MERLE et al au mont Ventoux [9]. 


\section{Formica gagates Latreille (FG).}

Fourmi dominante en sous-bois, dont on observe deux populations importantes. L'une est centrée au sud sur un nid avec couvain, sous une grosse pierre le plus souvent à l'ombre, enfoncée en terrain meuble sur le bord du chemin. Des ouvrières circulent sur le sol de la clairière dans la zone herbeuse voisine. Un second nid situé plus près de la source, à $17 \mathrm{~m}$ de celle-ci, sous une pierre, est abandonné; il s'agit peut être d'une localisation saisonnière de la $F$. gagates, ce nid étant abandonné en juillet au profit d'un emplacement à l'ombre. L'autre population est localisée à l'est dans le taillis qui abrite de très nombreuses ouvrières s'activant autour d'un petit chêne, le nid étant vraisemblablement très proche. II n'y a pas de nid dans la clairière, mais ceci pourrait tenir au comportement de $F$. gagates qui dispose toujours son nid au contact d'une racine de chêne ou à moins de quelques mètres $[2,9]$.

\section{Cataglyphis cursor (Fonscolombe) (CC).}

Quelques ouvrières courent rapidement dans la zone centrale de la clairière le matin, moins d'une heure après descente de la source; rien ne prouve qu'elles s'y soient trouvées auparavant. Aucun nid n'est repérable à moins de $30 \mathrm{~m}$, mais les ouvrières peuvent venir de très loin $(500 \mathrm{~m})$. Cette espèce nichait autrefois près de la source. Le MASNE et BONAVITA-COUGOURDAN [12] signalent, en effet, en 1970 deux orifices de sorties de nid qui n'existaient pas neuf mois auparavant.

\subsection{Espèces non trouvées sur place, autrefois présentes}

Myrmica scabrinodis Nyl. se trouve sous les pierres à $200 \mathrm{~m}$ sous bois. Les grandes espèces, Camponotus pilicornis Roger Var massiliensis (détermination Kuter) et Camponotus cruentatus (Latreille) nichaient à proximité de la source. On ne les y observe plus. Les petites espèces Leptothorax niger Forel et Leptothorax unifasciatus (Latreille) étaient également présentes; il est possible qu'elles s'y trouvent encore et qu'on ne les ait pas décelées. II en est de même de Myrmecina graminicicola (Latreille), petite espèce difficile à trouver en raison de ses mœurs semi-endogènes. Les perturbations apportées au milieu sont suffisantes pour expliquer ces absences, mais on ne peut pas exclure une radiosensibilité de ces espèces.

\subsection{Espèces trouvées sur place, autrefois absentes}

On remarque Tetramorium caespitum et Tetramorium semilaeve qui se sont introduits aussi bien en clairière que sous futaie. En lisière de la futaie on trouve Leptothorax angustulus, Diplorhoptrum fugax, Camponotus piceus et Dolichoderus quadripunctatus. Le nid des deux dernières espèces se situe peut être en dehors de la zone prospectée.

\subsection{Numération des sociétés}

Deux nids avec couvain sont identifiables dans la zone: un nid de Tetramorium caespitum (L.), trouvé sous un galet situé dans la zone herbeuse et un nid de Formica gagates Lat., trouvé sous une très grosse pierre enfoncée en sol meuble en bordure du chemin. Ceci ne représente que deux sociétés, mais la présence des nombreuses ouvrières témoigne de la proximité d'autres nids que nous ne nous sommes pas acharnés à localiser. Bien que toutes les pierres aient été retournées et remises en place, on a évité une exploration destructrice. Pour estimer le nombre de sociétés, on se basera donc sur l'existence de rassemblements d'ouvrières d'une même espèce et sur la notion de rayon d'action suffisamment court pour que la proximité du nid soit vraisemblable. 
On dénombre:

1 société de Tetramorium caespitum au moins (1 nid et présence étendue dans le quart sud-est),

1 société de Tetramorium semilaeve au moins (présence étendue dans le quart sud-est),

2 sociétés de Formica gagates ( 1 nid au sud, 1 société au nord),

1 société au moins de Pheidole pallidula (centre, nord et sud-ouest),

1 société de Leptothorax angustulus (litière au sud-ouest),

1 société de Diplorhoptrum fugax (litière au sud-ouest),

1 société de Crematogaster scutellaris au moins (chênes pubescents en bordure sud-ouest),

1 société de Plagiolepis pygmaea (taillis en bordure nord-est),

soit, au total, au moins 9 sociétés à moins de $25 \mathrm{~m}$ de la source. Ce chiffre est à comparer aux 27 sociétés recensées par Le MASNE et BONAVITA-COUGOURDAN [12] à l'intérieur d'un périmètre légèrement plus grand. II n'est pas possible de savoir si les fourmis qui courent sur la zone (Cataglyphis cursor, Camponotus piceus, Dolichoderus quadripunctatus) ainsi que Aphaenogaster subterranea dont on a pris seulement un mâle, nichent à l'intérieur de la zone inventoriée ou bien si elles proviennent de l'extérieur. Le nombre de sociétés pourrait donc être plus grand que 9 mais dans la meilleure hypothèse ce nombre est sûrement inférieur à 27 .

\subsection{Dominance}

Les deux espèces dominantes en 1969 et 1972 paraissaient être Formica gagates et Cataglyphis cursor [12]. Formica gagates s'est maintenu mais Cataglyphis cursor a été remplacé par Tetramorium caespitum et Tetramorium semilaeve qui semblent dominants avec Pheidole pallidula déjà présent en 1968. Crematogaster scutellaris semble fortement implanté localement, comme à l'origine.

\subsection{Recherches d'anomalies}

Tous les exemplaires recueillis sont morphologiquement normaux. Les différences importantes observées entre Tetramorium sont dans la limite des variations connues à l'intérieur du genre Tetramorium, c'est d'ailleurs une question encore débattue. Le comportement des ouvrières ne présente apparemment rien d'anormal et tous les individus observés ont la vivacité habituelle de leur espèce.

\section{DISCUSSIONS}

\subsection{Problèmes posés}

Le MASNE et BONAVITA-COUGOURDAN [12] signalaient déjà "que la faune des fourmis de la station choisie pour l'expérience n'était pas d'une extrême richesse" et d'autre part que la détection des effets éventuels de l'irradiation s'avérait difficile, d'autant plus que le repérage des nids sous couvert n'était pas toujours possible. Les conditions de l'inventaire initial n'étant pas reproductibles (disparition du piquetage, zone centrale remaniée 
en partie) il nous est apparu préférable de ne tester que la présence ou l'absence des espèces et d'inventorier les principales niches caractérisant ce biotope, d'où l'emploi de plusieurs méthodes de récolte et d'investigation en parallèle. Des omissions sont possibles, la zone étudiée (1350 $\mathrm{m}^{2}$ environ) étant importante et la recherche des nids difficile dans les taillis et dans les herbes. Nous nous sommes d'autre part limités, par nécessité, aux heures de plein jour entre 9 et $16 \mathrm{~h}$, ce qui empêche le recensement des espèces à préférence crépusculaire ou nocturne lorsque les nids ne sont pas accessibles (Myrmecina, par exemple). L'oubli de quelques petites colonies, trop discrètes pour avoir été observées, ne paraît pas susceptible, toutefois, d'altérer les conclusions auxquelles nous pouvons parvenir.

Nous devrons à cette fin discuter et opposer deux ordres de faits, concernant d'un côté les fourmis et de l'autre l'environnement. On sait, en effet, que les fourmis peuvent répondre à des modifications souvent minimes de leur environnement et l'on connaît leur faible radiosensibilité relative (par rapport, par exemple, à celle des mammifères). On sait d'autre part que l'irradiation peut affecter directement un écosystème en éliminant les espèces végétales les plus radiosensibles (on met de cette façon en évidence des effets de seuil) et entraîner ainsi indirectement le remplacement des associations végétales initiales par de nouvelles. Ces perturbations induisent, ensuite, des modifications de l'entomofaune associée. La question réellement posée est alors de savoir ce qui revient à chacune de ces composantes et, pour commencer, de savoir si on peut les isoler les unes des autres.

\subsection{Aspects radiobiologiques}

\subsubsection{Conditions dosimétriques}

La formule $I=1,46.10^{-10} C * E * P / D^{2}$ permet d'estimer en première approximation la dose délivrée en un point en milligrays par heure. Ainsi calculé, le débit de dose à la surface du sol en 1985 est compris entre $227 \mathrm{mGy} \cdot \mathrm{h}^{-1}$ au pied de la source et, environ $8 \mathrm{mGy} \cdot \mathrm{h}^{-1}$ à la limite de la clairière. La dose délivrée en un an est donc comprise entre 2000 Gy près de la source et 70 Gy à la lisière de la forêt. Mais les fourmis sont exposées au ras du sol où il faut tenir compte de la rétrodiffusion photonique et électronique. II faut, par ailleurs, prendre en compte des effets d'écrans dus au support métallique de la source ou à la végétation. C'est pourquoi la dose réelle a été estimée par des dosimètres radioluminescents. Les isodoses ainsi calculées en 1969 nous ont servi de base pour estimer les isodoses en 1985 (figures 1 et 2 ).

\section{Facteur d'écran}

Le premier nid comportant du couvain visible est situé sous un galet à $16,5 \mathrm{~m}$ du pied de la source. Le débit de dose y est réduit à $23 \mathrm{mGy} . \mathrm{h}^{-1}$ diminué de l'effet d'écran par le galet. Toutes les ouvrières capturées en nombre près de la source sont protégées par une pierre d'environ $10 \mathrm{~cm}$ d'épaisseur. Les sociétés de Pheidole pallidula et de Tetramorium caespitum et Tetramorium semilaeve s'enfoncent en terre en été pour échapper à 


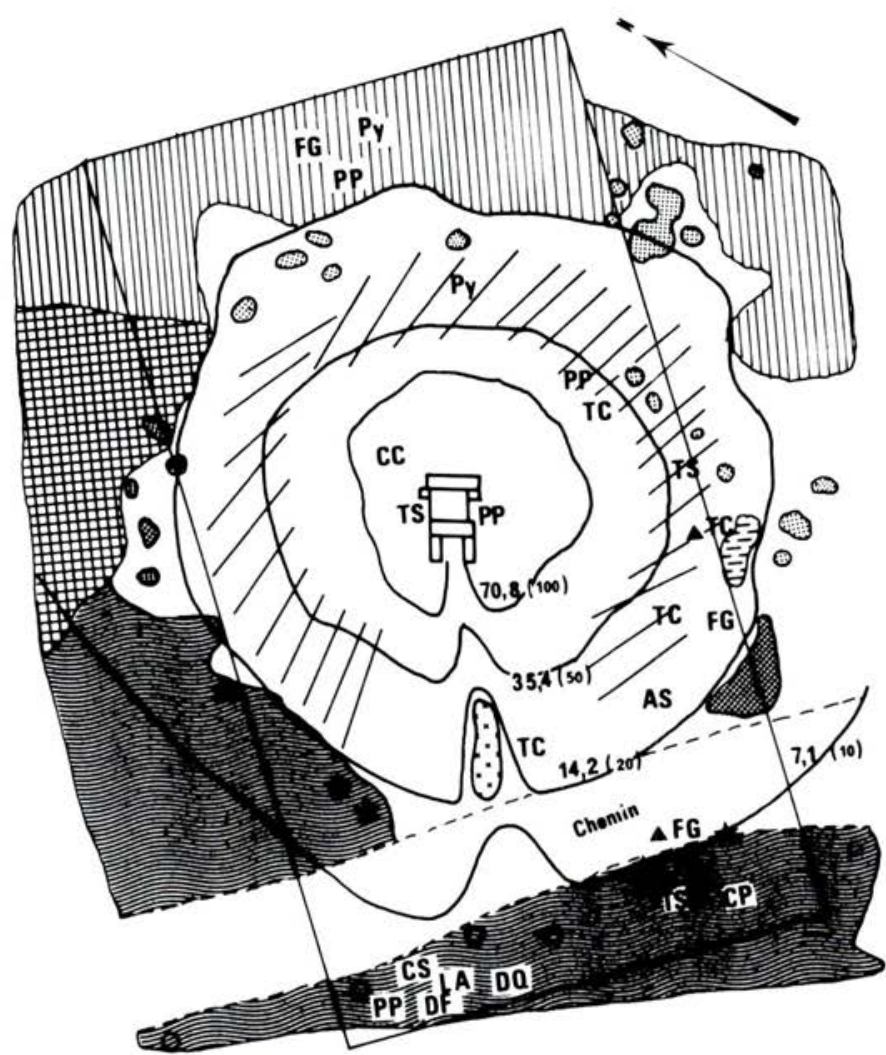

Fig. 2. - Végétation autour de la source du vallon des Charbonniers. Isodoses en 1969 (entre parenthèses) et en 1985 (caractères gras). Points de capture des fourmis en 1985.

AS Aphaenogaster subteranea (Latreille)

CC Cataglyphis cursor (Fonscolombe)

CP Camponotus piceus (Leach)

CS Crematogaster scutellaris (Olivier)

DF Diplorhoptrum fugax (Latreille)

DQ Dolichoderus quadripunctatus (Linné)
FG Formica gagates (Latreille)

LA Leptothorax angustulus (Nylander)

PP Pheidole pallidula (Nylander)

Py Plagiolepis pygmaea (Latreille)

TC Tetramorium caespitum (André)

TS Tetramorium semilaeve (André) $\because \because \quad$ Rosa pimpinellifolia

Zone herbacée

VID Teucrium - Hypericum)

Quercus pubescens

Sous-bois de Cornus mas

$\star \quad$ Chandelle d'arbres

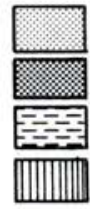

Rosmarinus officinalis

Phyllirea angustifolia

Phyllirea angustifolia (arbres morts)

Bois de Quercus ilex

Bois de Quercus ilex et Quercus pubescens

Bois de Quercus pubescens 
la chaleur et à la sécheresse ou à la lumière. Si l'on admet un facteur de réduction de dose de 2 à 4 sous une pierre de $10 \mathrm{~cm}$ d'épaisseur et de 2 pour $8 \mathrm{~cm}$ de terre ${ }^{\star}$, on est conduit à considérer que la dose effectivement cumulée par an est certainement inférieure à 1000 Gy.

\section{Facteur "temps d'exposition"}

Dans les conditions normales, les fourmis ne sont pas constamment en surface. Plusieurs facteurs sont susceptibles d'influer sur le temps d'exposition. Comme l'a montré DU MERLE [9] au mont Ventoux, certaines sociétés se déplacent en été de la clairière vers la forêt ou bien s'enfoncent dans le sol et reprennent le chemin inverse quand reviennent les conditions initiales. La dose reçue par certaines colonies au cours d'une année n'est donc pas nécessairement un simple multiple du débit de dose au point où elles sont découvertes.

La dose maximale que peut recevoir une fourmi doit, par ailleurs, être diminuée du temps pendant lequel la source est abaissée pour raison technique et en proportion de la durée de vie des fourmis. L'unité de temps à prendre en compte varie suivant les espèces et les castes. C'est ainsi que le mois convient pour les ouvrières et certaines reines alors que l'année sied à la reine de Plagiolepis pygmaea. On peut donc considérer que la dose effectivement reçue par un individu durant toute sa vie est, dans presque tous les cas, très inférieure à 1000 Gy (200 ou 300 Gy semblent une limite plus réaliste). Connaître ce paramètre permettrait de comparer les résultats obtenus in situ avec les données obtenues en laboratoire, mais cela semble fort difficile.

\section{Influence de la taille de l'insecte}

COLE et al (1979) démontrent que la taille de l'insecte irradié influe considérablement sur sa radiosensibilité estimée par l'effet létal (tableau I). Portés en coordonnées bilogarithmiques, les résultats s'ordonnent linéairement suivant une pente négative. La dose létale $50 / 24 \mathrm{~h}$ est donc une fonction de

TABLEAU ।

Radiorésistance de quelques insectes en fonction de leur poids.

Résultats obtenus à partir des expériences de COLE et al. [8].

\begin{tabular}{|l|r|r|}
\hline \multicolumn{1}{|c|}{ ESPĖCE } & \multicolumn{1}{|c|}{$\begin{array}{c}\text { Poids } \\
\text { (en mg) }\end{array}$} & $\begin{array}{c}\text { DL 50/24 h } \\
\text { (en Gy) }\end{array}$ \\
\hline Monomorium pharaonis L. (Fourmi pharaon) & 1,20 & 1900 \\
Pedes humanes humanes (Poux de corps) & 1,36 & 1800 \\
Cimes lectularius L. (Punaise des lits) & 4,37 & 1500 \\
Musca domestica L. (Mouche domestique) & 21,00 & 1100 \\
Thermobia domestica Pack (Lepisme domestique) & 21,28 & 980 \\
Blatella germanica L. (Blatte germanique) & 81,77 & 700 \\
Periplaneta americana L. (Blatte américaine) & 1148,00 & 500 \\
\hline
\end{tabular}

* Cette hypothèse s'accorde avec les résultats obtenus par J. LE GRAND et G. KENLAY (Commissariat à l'énergie atomique, DPS, Centre d'études nucléaires de Fontenay-aux-Roses), en juillet et septembre 1986. A partir des mesures dosimétriques effectuées par ces auteurs en plaçant les dosimètres en terre à $2,5,10$ ou $15 \mathrm{~cm}$ de profondeur et à 1,5 ou $4,4 \mathrm{~m}$ de la source on obtient une épaisseur moitié comprise entre 5,6 et $11,6 \mathrm{~cm}$.

VOL. $23-\mathrm{N}^{\circ} 1$ 
puissance du poids $P$ de l'insecte : $D_{50 / 24 h}=P^{-k}, k$ étant de l'ordre de 0,2. (Ils montrent ainsi que l'efficacité biologique du rayonnement gamma du cobalt $60(1,17$ et $1,33 \mathrm{MeV})$ varie dans le rapport de 4 à 1 quand on passe d'une grosse blatte Periplaneta americana à une très petite fourmi, Monomorium pharaonis. Pour expliquer cet effet de taille, on peut évoquer soit une diminution de l'EBR avec la taille, soit l'intervention de facteurs microdosimétriques (cette question est à l'étude). Mais à défaut de connaître l'explication de ce phénomène, on peut envisager d'appliquer aux espèces présentes sur le site de Cadarache la loi qui semble lier la dose létale 50/24 h au poids de l'insecte. On constate alors que Cataglyphis cursor, dont les ouvrières mesurent de 4,3 à $7,2 \mathrm{~mm}$, devrait être 1,15 à 1,30 fois plus radiosensible aux effets précoces que Tetramorium caespitum $(3,2$ à $4 \mathrm{~mm})$, Tetramorium semilaeve (2 à $3,2 \mathrm{~mm}$ ) et Pheidole pallidula $(1,1$ à $2 \mathrm{~mm}$ ). L'augmentation de la radiorésistance ainsi constatée, quand on passe d'un insecte de grande taille à un insecte plus petit, n'est pas considérable mais n'est peut être pas négligeable.

3.2.2. Etudes radioécologiques sur le terrain (tableau II).

TABLEAU ॥

Devenir de colonies de fourmis irradiées en conditions naturelles.

Comparaisons des données acquises à Cadarache avec les résultats de BROWER (1966) et CADWELL [5-6]

\begin{tabular}{|c|c|c|c|}
\hline & Source & $\begin{array}{l}\text { Débit de dose } \\
\text { mGy.h } h^{-1}\end{array}$ & $\begin{array}{c}\text { Réponse } \\
\text { d'une colonie }\end{array}$ \\
\hline $\begin{array}{l}\text { Pogonomyrmex occidentalis Cresson } \\
\text { Myrmicine }\end{array}$ & ${ }^{137} \mathrm{Cs}$ & 180 & $\begin{array}{l}\text { S'installe et } \\
\text { prospère }\end{array}$ \\
\hline Formica integra NYL Formicine & ${ }^{137} \mathrm{Cs}$ & 100 & Périclite \\
\hline $\begin{array}{l}\text { Tetramonium semilaeve Andre } \\
\text { Myrmicine }\end{array}$ & ${ }^{137} \mathrm{Cs}$ & $\begin{array}{c}229 \\
\text { (Cadarache) }\end{array}$ & $\begin{array}{l}\text { S'installe et } \\
\text { prospère }\end{array}$ \\
\hline $\begin{array}{l}\text { Tetramonium caespitum L. } \\
\text { Myrmicine }\end{array}$ & ${ }^{137} \mathrm{Cs}$ & $\begin{array}{l}23 \text { à } 40 \\
\text { (Cadarache) }\end{array}$ & $\begin{array}{l}\text { S'installe et } \\
\text { prospère }\end{array}$ \\
\hline $\begin{array}{l}\text { Plagiolepis pygmaea L. } \\
\text { Myrmicine }\end{array}$ & ${ }^{137} \mathrm{Cs}$ & $\begin{array}{c}229 \\
\text { (Cadarache) }\end{array}$ & $\begin{array}{l}\text { Se maintient } \\
\text { et prospère }\end{array}$ \\
\hline
\end{tabular}

CADWELL [6] montre que sous irradiation chronique par les rayons du césium 137 avec un débit de 180 mGy. $^{-1}$, une colonie de Pogonomyrmex occidentalis Cresson peut s'installer et prospérer. A l'inverse, BROWER [5] observe qu'une colonie de Formica integra Nyl ne supporte apparemment pas 100 mGy. $\mathrm{h}^{-1}$ du même rayonnement et périclite. II n'exclue pas une explication par la baisse des ressources alimentaires consécutive à la modification de l'environnement. Le débit de dose est, sur le site de Cadarache, compris entre 227 mGy. $\mathrm{h}^{-1}$ au pied de la source et environ $8 \mathrm{mGy} \cdot \mathrm{h}^{-1}$ à la limite de la clairière. Trois espèces seulement résistent à ces conditions : Tetramorium semilaeve Andre et Pheidole pallidula ( $\mathrm{Nyl})$ qui sont exposées au débit de dose maximale et Tetramorium caespitum (L.) dont le nid à $15 \mathrm{~m}$ de la source ne subit pas plus de $23 \mathrm{mGy}^{-\mathrm{h}^{-1}}$ et les individus les plus proches de la source, 40 mGy.h ${ }^{-1}$. Ces résultats sont comparables aux deux précédents car ils résultent de débits de dose relativement proches comparés aux débits de dose, parfois considérables, qu'utilisent les expérimentateurs travaillant en laboratoire. 


\subsubsection{Etudes d'irradiations en laboratoire (tableau III).}

GANO [11] démontre que chez Pogonomyrmex oweheii Cole recevant 35 à 2680 Gy sous un débit compris entre 275 et 420 Gy. $\mathrm{h}^{-1}$ de rayonnement gamma du césium 137, le taux de mortalité est égal à celui de populations témoins. A l'inverse, TOROSSIAN [22] montre que, pour des doses allant de 125 à 1500 Gy délivrées avec un débit de dose de $624 \mathrm{~Gy} \cdot \mathrm{h}^{-1}$ par les rayons gamma du cobalt 60 , la durée de vie est diminuée chez Dolichoderus quadripunctatus (L.). Pour la dose la plus faible, 125 Gy, le temps nécessaire pour atteindre la DL 50 passe à 18 jours dans les colonies d'ouvrières quand elle était de 30 jours chez les témoins et la DL100 s'obtient en 45 jours au lieu de 78,7 jours. A 500 Gy le temps nécessaire pour atteindre la DL 50 n'est plus que 16 jours et la DL100 s'obtient en 40 jours environ. Les reines sont beaucoup plus résistantes, leur durée de vie passant de 172 jours chez les témoins à 112 jours pour 500 Gy; elles manifestent, par contre, une baisse très nette de la fécondité, les œufs n'étant pas viables. Les ouvrières pondeuses sont complètement stérilisées à 125 Gy. Dans les mêmes conditions, la consommation d'oxygène des ouvrières est augmentée de façon significative [22]. Elle passe de $1,8 \mathrm{ml} \cdot \mathrm{h}^{-1}$ chez les témoins à 2,2 pour 500 Gy et 2,7 pour 1000 Gy.

MENHINICK et CROSSLEY [14] exposent des ouvrières d'âge inconnu de Crematogaster lineolata Say à différentes doses de rayonnement gamma. Les sources de cobalt 60 utilisées ont un débit $1398 \mathrm{~Gy}^{-\mathrm{h}^{-1}}$ pour une gamme de dose administrée de 10, 20 et 40 Gy. Le débit de dose est $13980 \mathrm{~Gy} \cdot \mathrm{h}^{-1}$ pour les doses administrées supérieures à 40 Gy. A 10 Gy, la ciurée de vie moyenne des fourmis n'est pas affectée. A partir de 40 Gy, elle décroît progressivement et passe de 82,7 jours, chez les individus témoins, à 18 jours chez les individus irradiés à 160 Gy et à 3,3 jours chez les individus irradiés à 1280 Gy. Aucune ouvrière ne survit à la dose de 5120 Gy. Les doses utilisées atteignent donc celles utilisées pour Pogonomyrmex oweheii, mais les débits de dose sont considérablement plus élevés.

PASSERA [15] soumet à une irradiation gamma de 700 Gy (cobalt 60), des femelles de Plagiolepis pygmaea Latreille et deux de ses parasites sociaux Plagiolepis xene St. ou Plagiolepis grassei Le Masne. Ces femelles sont irradiées en présence de 100 ouvrières en utilisant la même source que TOROSSIAN [22] (624 Gy.h $\left.\mathrm{h}^{-1}\right)$. Dans ces conditions, la durée de vie d'une reine, qui est dans la nature de quelques années, n'est plus que de 39 à 61 jours. Les parasites sociaux sont un peu plus sensibles.

COLE et al. [8] irradient des ouvrières de Monomorium pharaonis (L.) au moyen de cobalt 60 avec un débit de dose de 240 ou 600 Gy.h $\mathrm{h}^{-1}$. Ils évaluent ainsi la dose nécessaire pour tuer $50 \%$ ou $100 \%$ des individus en $24 \mathrm{~h}$.

Les différents résultats obtenus en laboratoire sont rassemblés dans le tableau III. Dans la même colonne sont associés les durées de vie moyenne et les temps nécessaires pour obtenir la DL50 lorsqu'on ne connaît pas les durées de vie. Pour pouvoir dire que ces quantités sont comparables, il faut évidemment présumer que les courbes de mortalité sont semblables dans les deux cas. (Les indications des auteurs cités ne permettent pas d'en être certain). 
G.H. Perrault, R. CAstet

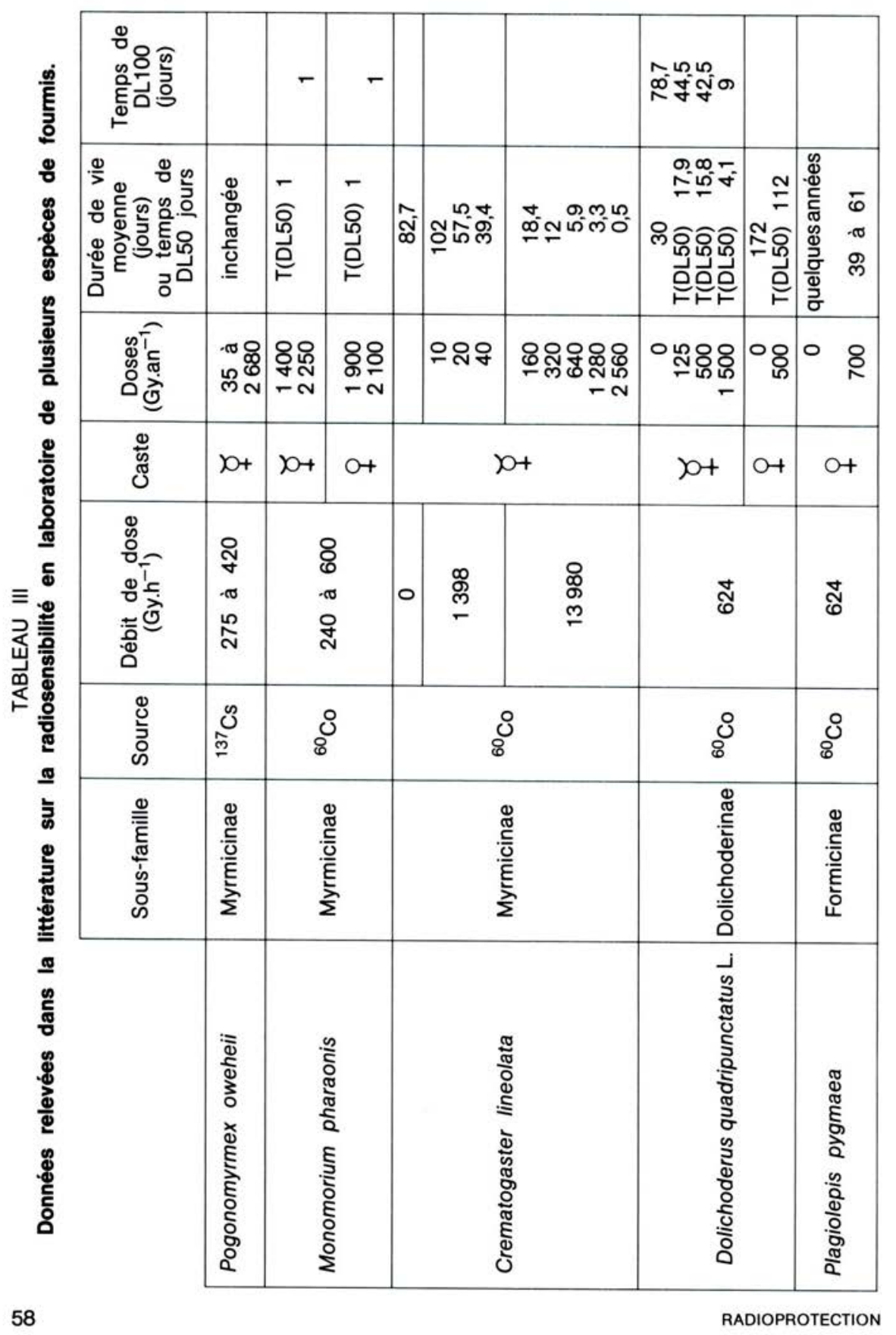




\section{Comparaison de nos résultats avec la littérature}

Les principaux résultats acquis en laboratoire sont résumés dans le tableau III. On constate que Pogonomyrmex oweheii se révèle très résistant à des doses allant jusqu'à 2680 Gy avec un débit élevé. Mais il serait hasardeux d'établir une échelle de valeur de la radiorésistance des quatre autres fourmis. Peut-on dire, en effet, qu'une espèce dont la durée de vie après une certaine irradiation passe de 82 à 18 jours est plus radiosensible qu'une espèce dont la durée de vie passe de 30 jours à 18 jours, surtout si les individus de la première sont des "tout venant" d'âge quelconque et donc très probablement en majorité des ouvrières âgées, alors que les individus de la seconde sont issus d'un nid sortant d'hibernation ? Ces études même correctement quantifiées seront toujours difficilement comparables.

\subsubsection{Radiorésistance et habitudes alimentaires (tableaux II et III)}

Nous entendons ici faire une remarque sur une coïncidence, peut-être fortuite en raison du peu de données disponibles, mais qui pourrait prendre un intérêt particulier si elle se confirmait. On note, en effet, que les seules espèces apparemment radiorésistantes appartiennent à la sous-famille des Myrmicinae (Pogonomyrmex occidentalis Cresson et $P$. oweheii Cole). Ce sont des fourmis rustiques principalement granivores et couramment appelées "Harvester ants" aux USA. Elles sont bien adaptées aux déserts nord américains. Or, les seules espèces implantées dans un rayon d'une vingtaine de mètres autour de la source, sur le site de Cadarache, sont les Myrmicinae (Tetramorium caespitum (L.) et $T$. semilaeve Andre, Pheidole pallidula (Nyl.). Ces trois espèces sont omnivores, mais il arrive que certaines colonies se spécialisent dans l'exploitation des graines (BERNARD, 1968).

A l'inverse, Crematogaster lineoleta et Monomorium pharaonis qui appartiennent à deux genres de la même sous-famille de Myrmycinae sont relativement radiosensibles. La radiorésistance n'est donc pas un critère chez les Myrmicinae. II semblerait, par contre, que la radiorésistance se retrouve chez les espèces qui possèdent la capacité de résister à des conditions environnementales très rudes. On retrouve l'observation faite à Cadarache par FABRIES [10] puis par BATTESTI-MARCHESE [1] à propos des végétaux entourant la source. II semble, en effet, à ces auteurs que les ubiquistes, qui s'adaptent aux régions et aux climats les plus divers, sont les plus radiorésistantes alors que les euroasiatiques sont les plus radiosensibles en raison de fortes variations des conditions du milieu qu'elles ne tolèreraient pas dans leurs domaines écologiques naturels.

On ne sait pas, pour le moment, si la radiorésistance se retrouve dans d'autres sous-familles de fourmis, mais, si on extrapolait les conclusions précédentes, on pourrait s'attendre à ce que Cataglyphis cursor, qui appartient à un genre de Formicinae possédant de nombreux représentants sous des climats arides et désertiques, soit radiorésistant puisqu'il est capable de subir dans la nature un environnement physique très hostile. Or, Le MASNE et BONAVITA-COUGOURDAN [12] ont constaté la présence, 11 mois après irradiation, de deux orifices de sortie du nid de cette espèce tout près de la source. Ceci implique une certaine radiorésistance et on peut penser que la disparition de Cataglyphis cursor est à mettre sur le compte, non pas 
d'une radiosensibilité spécifique directe, mais sur celui d'une sensibilité purement écologique à la modification de l'environnement secondaire à l'irradiation de l'écosystème. Nous dirions alors qu'il s'agit d'une “radioécosensibilité" et non pas d'une radiosensibilité de l'espèce.

\subsection{Aspects écologiques}

Les travaux de FABRIES [10] montrent bien que l'irradiation de la clairière autour de la source de Cadarache a eu pour effet de modifier notablement les phytocénoses préexistantes. Les observations de BATTESTI-MARCHESE [1] confirment cette évolution et conduisent à constater que l'écosystème a évolué depuis l'origine vers un état d'équilibre. Toute aussi importante est la remarque selon laquelle les espèces végétales dominantes se remplacent le long du gradient d'irradiation selon une séquence qui reproduit celle que l'on trouve le long d'un gradient environnemental naturel. On peut donc se demander si les modifications de l'environnement, abstraction faite de l'irradiation qui les a produites, ne pourraient pas, à elles seules, expliquer l'évolution de la faune myrmécologique. II nous faut donc analyser les conséquences, pour les fourmis, du choix du site d'expérimentation particulier que constitue une clairière.

Selon DU MERLE et al [9] une clairière ne constitue pas, pour les fourmis, un écosystème isolé. L'écosystème comprend, en fait, trois zones concentriques: une clairière centrale, une lisière de quelques mètres de largeur formée d'un taillis de faible hauteur, et la forêt périphérique. Certaines fourmis peuvent migrer d'une zone à l'autre en fonction de la saison alors que d'autres restent dans la même zone toute l'année. Or, sur le site de Cadarache, l'irradiation chronique, comme nous l'avons signalé en 2.1., a appauvri le couvert végétal essentiellement dans la clairière qui s'est légèrement agrandie par rapport à celle d'origine. Le site initial est donc modifié mais n'a pas changé de nature puisqu'il s'agit encore du même type d'écosystème. Les arbres qui existaient en périphérie ont disparu ou subsistent à l'état de chandelle. Cet appauvrissement du couvert végétal, qui est un facteur contrôlant l'ambiance thermohygrométrique et l'ensoleillement, a pu inciter certaines espèces à émigrer, d'autant plus que la faune entomologique associée aux fourmis y est devenue très paurre. Dans les quelques mètres de largeur de la lisière (au sens de DU MERLE) se manifestent les derniers signes de souffrance végétale les plus évidents (chétivité, malformations et effets de masque) et la variété de la faune entomologique qui s'y trouve indique qu'on a atteint la limite d'action efficace du rayonnement. Cette variété est banale: c'est l'une de celles que l'on peut s'attendre à trouver en lisière dans les conditions normales du mois de juillet. On peut donc penser que l'écosystème primitif, bien que modifié, persiste en tant que tel et reste soumis aux lois écologiques de l'alternance saisonnière. Mais il faudrait, pour le prouver, comparer cette situation estivale avec celles du printemps et de l'automne. On regrettera, cependant, que la source ait été placée au centre de la clairière et non pas en situation excentrique, ce qui aurait permis de dissocier l'effet de lisière de celui de limite d'atteinte radiologique.

Le fait que de nombreuses espèces n'apparaissent qu'à partir de la lisière n'est pas nécessairement imputable à un effet direct de l'irradiation. 
C'est ainsi que l'on trouve à quelques mètres, en forêt, des espèces qui n'ont vraisemblablement jamais niché au centre de la clairière, bien que certaines puissent s'y aventurer pour chercher leur nourriture. C'est sûrement le cas de Crematogaster scutellaris, de Leptothorax angustulus et Dolichoderus quadripunctatus. II en est de même de Formica gagates qui fait son nid au contact, ou non loin, d'une racine de chêne; c'est une espèce de sousbois, volontiers arboricole, mais dont le rayon d'action est important et il est normal de la trouver loin de son nid.

Plagiolepis pygmaea est, par contre, une espèce omnivore que l'on pourrait s'attendre à trouver sous les pierres de la clairière. Ne s'y trouve-t-elle plus en raison d'une certaine radiosensibilité ou à cause de la disparition des pucerons qu'elle lèche pour se nourrir ? En fait, nous savons par les travaux de DU MERLE et al [9] que cette espèce migre de la clairière vers la forêt quand arrivent les jours chauds et secs de l'été. II faudrait donc faire une nouvelle enquête au mois de mai pour savoir si cette migration se produit encore sous irradiation.

A l'inverse, Cataglyphis cursor se cantonne aux formations xérophiles méditerranéennes et était bien à sa place dans la clairière à romarin où existaient deux sociétés avant l'irradiation. Les conditions ont ensuite changé: le romarin a disparu, remplacé par des végétaux bas et le sol a été assez piétiné. On peut penser que les grandes fourmis insectivores n'ont plus trouvé, après cet appauvrissement du biotope, la quantité de nourriture qui leur était nécessaire bien qu'elles soient capables de chasser très loin de leur nid. Le même raisonnement peut s'appliquer à Camponotus cruentatus dans la mesure où il nidifiait à découvert avant l'installation de la source.

D'autres espèces sont connues pour leur ubiquité. II en est ainsi de Pheidole pallidula, codominant dans la clairière, mais présent en forêt sèche; c'est une espèce méditerranéenne qui affectionne la chaleur et se développe aussi bien sous bois que sur un causse bien qu'elle semble préférer les terrains silicicoles [2].

Tetramorium caespitum s'adapte aux conditions difficiles; c'est une fourmi qui peut établir des nids au bord des routes. A la suite de leurs travaux au mont Ventoux, DU MERLE et al [9] pensent que Tetramorium caespitum s'installe lorsque les conditions deviennent difficiles et que la concurrence diminue parce que cette fourmi est rustique mais peu compétitive; elle occupe donc le terrain laissé libre par des espèces plus exigeantes, c'est ici le cas. II n'est pas, par ailleurs, exceptionnel de voir Tetramorium semilaeve voisiner avec Tetramorium caespitum malgré des préférences écologiques différentes de ces deux espèces (BERNARD, [2, 3]).

Les deux espèces de Tetramorium et Pheidole pallidula sont donc, dans la nouvelle clairière, dans un de leurs biotopes d'élection en l'absence d'irradiation.

On sait très bien enfin que les sociétés de fourmis ne subsistent pas indéfiniment au même endroit bien qu'on en ait parfois observées pendant 20 ou 30 ans. La moindre variation infligée à leur biotope soit du fait de 
modifications naturelles de l'écosystème, soit du fait de l'homme peut entraîner la décadence des colonies. Les fourmis sont, en particulier, très sensibles aux variations de l'ensoleillement résultant du remplacement d'une communauté végétale par une autre [13]. De telles perturbations qui sont, en fait, indépendantes de l'irradiation se sont produites pendant 16 ans sur le site de Cadarache (piétinement, pose de grillages et de bacs). Les interventions humaines répétées ont ainsi, à elles seules, pu entraîner le départ de Cataglyphis cursor et de Camponotus cruentatus et leur remplacement par des Tetramorium.

\section{CONCLUSION}

Etant données les conditions ponctuelles de l'observation, il serait hasardeux d'en tirer une conclusion générale sur l'état du peuplement myrmécologique de l'écosystème forestier sous irradiation gamma chronique. Cependant, nous pouvons faire les constatations importantes qui suivent. Sous l'effet direct ou indirect des rayonnements ionisants, le nombre des sociétés a regressé mais le nombre des espèces recensées est resté approximativement le même, certaines grandes espèces insectivores ayant été remplacées par de petites espèces omnivores plus rustiques. Cette substitution est particulièrement évidente dans un rayon de 15 à $20 \mathrm{~m}$ autour de la source et s'explique par la modification de l'environnement physique lié à l'amenuisement du couvert végétal et par l'appauvrissement des ressources alimentaires; elle implique, également, une radiorésistance relativement importante de trois espèces de Myrmicinae, dont deux espèces nouvellement introduites, qu'on ne peut manquer de rapprocher des deux cas connus de résistance chez des Myrmicinae granivores. Cette comparaison suggère que la possibilité qu'ont certaines espèces de s'adapter à un environnement naturel sévère leur confère une radiorésistance plus élevée ainsi qu'une facilité à s'adapter aux modifications écologiques induites par le rayonnement ("radioécorésistance").

On ne note, d'autre part, aucune transformation ni morphologique, ni comportementale, mais on ne peut exclure une radiosensibilité spécifique des espèces éliminées, même si des raisons écologiques semblent suffir pour expliquer leur départ.

A la question de savoir ce qui revient à l'irradiation d'une part, aux modifications de l'environnement induites par l'irradiation d'autre part, on peut apporter des éléments de réponse par comparaison avec l'action du rayonnement sur les phytocénoses. Les espèces végétales dominantes se remplacent, en effet, le long du gradient d'irradiation selon une séquence semblable à celle que l'on observe le long du gradient environemental naturel. Ce gradient traduit ici le passage de la clairière à la forêt et à sa litière et on sait l'importance qu'il revêt pour la distribution des espèces. La transition clairière-forêt s'est déplacée sous irradiation et correspond actuellement, là où il est le mieux défini en bordure du Quercetum pubescentis à des débits de dose compris entre 8 et $4 \mathrm{mGy} \cdot \mathrm{h}^{-1}$. Cette transition coïncide avec la limite des effets les plus visibles du rayonnement sur les végétaux ainsi qu'avec la réapparition d'une faune entomologique variée quoique peu 
abondante et particulièrement de la faune myrmécologique. II est donc difficile de bien séparer l'effet de lisière de celui de la réduction de dose due à l'éloignement de la source. Quoi qu'il en soit, après le bouleversement qui a suivi l'installation de la source, une nouvelle structure s'est établie avec de nouvelles fonctions. Celle-ci est moins complexe que celle de départ, mais elle semble actuellement en équilibre.

Dans les conditions expérimentales choisies à Cadarache, l'irradiation constitue finalement un stress physique comme un autre. II ne détruit pas l'écosystème clairière - forêt dont l'involution progressive semble limitée par la sélection d'espèces animales ou végétales résistantes. Ce mécanisme s'apparente à la réaction d'adaptation d'un écosystème à des conditions physiques devenues difficiles, par exemple à la sécheresse.

C'est un constat plutôt optimiste.

\section{BIBLIOGRAPHIE}

[1] BATTESTI-MARCHESE M.J. Etat de la phytocénose terrestre du site irradié de plein air de Cadarache après 14 ans d'irradiation chronique. DEA Aix-Marseille III, $43 \mathrm{p}$.

[2] BERNARD F. Les fourmis d'Europe occidentale et septentrionale. Paris: Masson, 1968.

[3] BERNARD F. Les fourmis et leur milieu en France méditerranéenne. Paris: Le Chevalier, 1983.

[4] BIGOT L., GRAUBY A., POINSOT N., ROUGON C.D., TCHERNIA C. Influence d'une irradiation gamma chronique sur la faune et la microflore d'un écosystème forestier à Cadarache. Radioprotection, 1973, 8 (4), 243-256.

[5] BROWER J.H. Behavioral changes in an ant colony exposed to chronic gamma irradiation. Am. Midl. Nat., 1966, 75 (2) 530-534.

[6] CADWELL L.L. Colony formation of the western harvester ant in a chronic gamma radiation field. Am. Midl Nat., 1973, 89 (2) 446-448.

[7] CASTET R. Evolution des activités microbiennes dans un écosystème méditerranéen soumis à une irradiation gamma chronique. Etude particulière des transferts azotés par marquage isotopique. Thèse de Docteur-ingénieur, Université St-Jérôme, Marseille, 1986.

[8] COLE M.M., La BRECQUE C.C., BURDEN G.S. Effects of gamma radiation on some insects affecting man. Econ. Entomol., 1979, 52 (3) 448-450.

[9] DU MERLE P., JOURHEUIL P., MARRO J.P., MAZET R. Evolution saisonnière de la myrmécofaune et de son activité prédatrice dans un milieu forestier: les interactions clairière-lisière-forêt. Ann. Soc. Ent. Fr (NS), 1978, 14 (2) 147-157.

[10] FABRIES M. Quelques aspects écologiques et biogéographiques de l'irradiation chronique d'une phytocénose terrestre de type méditerranéen. Rapport CEA-R4300, 1972.

[11] GANO K.A.Mortality of the harvester ant (Pogonomyrmex owyheei) after exposure to ${ }^{137} \mathrm{Cs}$ gamma radiation. Ent. Soc. Amer, 1981, 10 (1), 39-44.

VOL. $23 \cdot \mathrm{N}^{\circ} 1$ 
[12] Le MASNE G., BONAVITA-COUGOURDAN A., Premiers résultats d'une irradiation prolongée au césium sur les populations de fourmis en Haute-Provence. Ekologia Polska, 1972, 20 (14) 129-144.

[13] LORBER B.E. Action de la végétation sur la dynamique d'une colonie polycalique de Coptoformica exsecta Nylander dans les conditions naturelles. Entomologiste, 1986, 42 (1) 27-38.

[14] MENHINICK E.F., CROSSLEY D.A. Radiation sensitivity of twelve species of arthropods. Ann. Ent. Soc. Amer. 1969, 62 (4) 711-717.

[15] PASSERA L. Action des radiations gamma sur la fourmi Plagiolepis pygmaea Latreille et sur deux de ses parasites sociaux: comparaison de leur longétivité. C.R. Acad. Sci. Paris, D, 1971, 273, 1502-1505.

[16] POINSOT-BALAGUER N. Réponse des communautés de Collemboles à l'irradiation gamma chronique d'une forêt méditerranéenne. Rev. Ecol. Biol. Sol, 1976, 13 (2) 365-379.

[17] ROUGON D. Etude écologique des coléoptères d'une chênaie soumise à une irradiation gamma chronique. Thèse de Docteur spécialiste, Faculté de Marseille, 1970.

[18] SAAS A., BOVARD P. GRAUBY A. The effects of chronic gamma irradiation on decay of oak (Quercus pubescens Will D.) and dogwood (Cornus mas. L.) leaves and subjacent litter. Radiat. Botany, 1975, 15, 141-151.

[19] TABONE E., POINSOT-BALAGUER N. Dynamique de la matière organique dans un sol de forêt méditerranéenne (chênes verts, Quercus ileus L., chênes blancs Quercus pubescens W.) soumis à une irradiation gamma chronique. Pedologia (à paraître).

[20] TOROSSIAN C. CAUSSE R. Effets des radiations gamma sur la fertilité et la longévité des colonies de Dolichoderus quadripunctatus. In: Isotopes and radiation in entomology. Vienne: AIEA, 1968, 155-164.

[21] TOROSSIAN C. Effets des radiations alpha et gamma sur la résistance et la longétivité d'ouvrières orphelines de la fourmi Dolichoderus quadripunctatus. C.R. Acad. Sc. Paris, 1970, 271, 987-990.

[22] TOROSSIAN V. Modifications de la consommation d'oxygène de la fourmi Dolichoderus quadripunctatus exposée à des irradiations gamma de forte puissance. C.R. Soc. Biol. Toulouse, 1978, 172, 542-545. 